\title{
High-Velocity Outflow in the Extended Emission-Line Region of the Seyfert Galaxy NGC 7319
}

\author{
Kentaro Aoki \\ Astronomical Data Analysis Center, National Astronomical Observatory \\ of Japan, Mitaka, Tokyo, 181, Japan
}

\section{Introduction}

NGC 7319 is a member of the compact group of galaxies known as Stephan's Quintet. The systemic velocity $6740 \mathrm{~km} \mathrm{~s}^{-1}$ gives a distance of $90 \mathrm{Mpc}\left(H_{0}=75\right.$ $\mathrm{km} \mathrm{s}^{-1} \mathrm{Mpc}^{-1}$ ) for NGC 7319. This galaxy has a tail and/or ring which suggests interaction with a neighboring pair of galaxies, NGC 7318A and B (e.g., Arp 1973). Durret \& Warrin (1990) and Durret (1994) performed optical long-slit spectroscopy and found an extended emission-line region (EELR) in NGC 7319. They detected highly excited gas over $12 \prime .5$ along $P A=61^{\circ}$ and $10^{\prime \prime}$ along $P A=151^{\circ}$. Apart from these works, there have not been any studies on the velocity field and excitation of the gas in the EELR of NGC 7319. More details were published by Aoki et al. (1996).

\section{Observations}

We made observations with the Cassegrain spectrograph attached to the $1.88-\mathrm{m}$ telescope at Okayama Astrophysical Observatory in 1994 October and November. The 1".8 width slit was set to seven different positions. Except for one position, the dispersion was $4.8 \AA$ pixel $^{-1}$ and wavelength coverage was $4600-$ $7000 \AA$. We obtained higher-dispersion spectra $\left(1.2 \AA\right.$ pixel $\left.^{-1}\right)$ at $P A=27^{\circ}$ over the spectral ranges $4800-5400 \AA$ and $6400-7000 \AA$.

\section{Results}

The EELR extends $10^{\prime \prime}(4.5 \mathrm{kpc})$ south-southwest from the nucleus, while in other directions it is less extended (Fig. 1a). The [O III] emission distribution agrees with the radio continuum image by van der Hulst \& Rots (1981). Both are elongated similarly toward the southwest direction.

The velocity field of $[\mathrm{O}$ III $] \lambda 5007$ is plotted on a two-dimensional map in Fig. 1b. Figure $1 \mathrm{~b}$ clearly shows that the ionized gas in the south-southwest region is blueshifted from the systemic velocity $\left(6740 \mathrm{~km} \mathrm{~s}^{-1}\right)$ by $300 \mathrm{~km} \mathrm{~s}^{-1}$, and the radio jet corresponds to this blueshifted region. Several kinematically distinct components in line profiles are also recognized.

The EELR spectrum yields [O III] $\lambda 5007 / \mathrm{H} \beta>10$, [O I] $\lambda 6300 / \mathrm{H} \alpha=0.17$, $[\mathrm{N}$ II $] \lambda 6583 / \mathrm{H} \alpha=0.79$, and $[\mathrm{S} \mathrm{II}] \lambda \lambda 6716+6731 / \mathrm{H} \alpha=0.69$, which places this spectrum in the 'Seyfert' region of diagnostic diagrams (Veilleux \& Osterbrock 

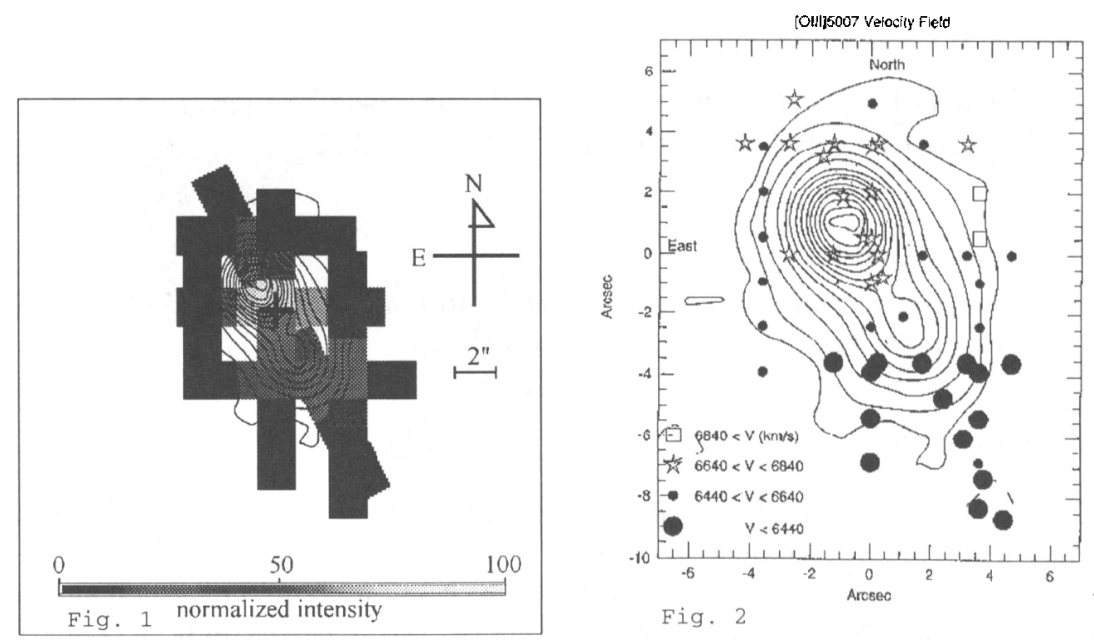

Figure 1. Left: $[\mathrm{O}$ III] $]$ 5007 emission intensity distribution. The optical continuum peak is indicated by the cross. Contours of the $20-\mathrm{cm}$ radio image by van der Hulst \& Rots (1981) are superposed. Right: [O III $] \lambda 5007$ velocity field. The different symbols represent the different velocity bins. Contours of the 20 -cm radio image by van der Hulst \& Rots (1981) are superposed. In both maps, north is up and east is to the left.

1987). The electron density of the nucleus and the EELR are estimated from [S II] doublet ratio as $300 \mathrm{~cm}^{-3}$ and $600 \mathrm{~cm}^{-3}$, respectively.

\section{Discussion}

The observations noted in the previous section indicate a nuclear high-velocity outflow in the EELR of NGC 7319. Besides the complicated line profiles, a relatively high density and a small volume-filling factor $\left(\sim 10^{-4}-10^{-5}\right)$ suggest the outflowing gas would be comprised of many small filaments or blobs which are compressed by interaction of the outflow with interstellar matter.

\section{References}

Aoki, K., Ohtani, H., Yoshida, M., \& Kosugi, G. 1996, AJ, 111, 140.

Arp, H.C. 1973, ApJ, 183, 411.

Durret, F., \& Warrin, F. 1990, A\&A, 238, 15.

Durret, F. 1994, A\&AS, 105, 57.

van der Hulst, J. M., \& Rots, A.H. 1981, AJ, 86, 1775.

Veilleux, S., \& Osterbrock, D.E. 1987, ApJS, 63, 295. 